\title{
Musashi-2 is a novel regulator of paclitaxel sensitivity in ovarian cancer cells
}

\author{
JEONGSUK LEE $^{1 *}$, SUNGKWAN AN $^{1 *}$, YEONG MIN CHOI $^{1,2}$, JUNWOO LEE $^{1}$, KYU JOONG AHN ${ }^{3}$, \\ JAE HO LEE ${ }^{4}$, TAE JIN KIM ${ }^{5}$, IN-SOOK AN ${ }^{2}$ and SEUNGHEE BAE ${ }^{1}$
}

\begin{abstract}
${ }^{1}$ Research Institute for Molecular-Targeted Drugs, Department of Biological Engineering, Konkuk University, Seoul 05029;
${ }^{2}$ Gene Cell Pharm Corporation, Cheongju-si, Chungcheongbuk-do 28160; ${ }^{3}$ Department of Dermatology, Konkuk University School of Medicine, Seoul 05030; ${ }^{4}$ Laboratory of Molecular Oncology, Cheil General Hospital and Women's Healthcare Center, Dankook University College of Medicine, Seoul 04619; ${ }^{5}$ Department of Obstetrics and Gynecology, Cheil General Hospital and Women's Healthcare Center, Dankook University College of Medicine, Seoul 04619, Republic of Korea
\end{abstract}

Received June 24, 2016; Accepted August 22, 2016

DOI: 10.3892/ijo.2016.3683

\begin{abstract}
As few prognostic markers and symptoms have been identified, ovarian cancer is typically diagnosed at an advanced stage, and a majority of patients will relapse and develop resistance to anticancer drugs such as paclitaxel. Musashi-2 (MSI2) is a regulator of gene translation and functions as an oncogenic protein and a marker of poor prognosis in various types of cancer. However, the biological and clinical significance of MSI2 in ovarian cancer remains unclear. Using a tissue microarray-based assay, we demonstrated that MSI2 was highly expressed in advanced, serous ovarian cancer tissues. In addition, MSI2-overexpressing ovarian cancer cells exhibited increased viability, proliferation and growth. We found that MSI2 was overexpressed in paclitaxel-resistant ovarian cancer SKOV3-TR cells but not in paclitaxel-sensitive cell lines. The loss of MSI2 expression in lentivirus-mediated stable MSI2 knockdown SKOV3-TR cells impaired paclitaxel resistance as determined using cell viability and apoptosis assays. In contrast, lentivirus-mediated MSI2 overexpression promoted the development of paclitaxel resistance in paclitaxel-sensitive ovarian cancer cells. The results of the present study are the first to demonstrate that MSI2 is a valuable marker of advanced, serous ovarian cancer and that MSI2 plays an important role in paclitaxel resistance.
\end{abstract}

Correspondence to: Dr Seunghee Bae, Research Institute for Molecular-Targeted Drugs, Department of Biological Engineering, Konkuk University, 120 Neungdong-ro, Gwangjin-gu, Seoul 05029, Republic of Korea

E-mail:sbae@konkuk.ac.kr

*Contributed equally

Key words: Musashi-2, paclitaxel, drug resistance, ovarian cancer cells, apoptosis, cytotoxicity

\section{Introduction}

The Musashi family of proteins comprises two evolutionarily conserved RNA-binding proteins: Musashi-1 (MSI1) and Musashi-2 (MSI2). MSI1 plays a pivotal role in stem cell maintenance $(1,2)$, whereas MSI2 is a recently identified oncogenic protein (3). MSI2 is frequently overexpressed in various types of human cancers and is associated with disease progression. It is overexpressed in patients with acute myeloid leukemia (AML) and chronic myeloid leukemia (CML), and MSI2 expression in AML and CML is directly correlated with a poor clinical prognosis $(4,5)$. Consistent with these findings, another report has demonstrated that elevated levels of MSI2 expression correlate with poor prognosis in patients with B-cell acute lymphoblastic leukemia (6). In addition, MSI2 is highly expressed in hepatocellular carcinoma (HCC) tissues but not in its corresponding non-tumorous tissues and that MSI2 overexpression correlates with highly invasive HCC (7). A recent study has confirmed that colorectal adenocarcinomas express high levels of MSI2 and that MSI2 functions as an oncogenic protein by promoting intestinal cell transformation (3). A subsequent study has demonstrated that the oncogenic function of MSI2 depends on its own structural feature, i.e., the presence of an RNA-recognition motif (8). This motif binds to specific cancer-associated target mRNAs, including the tumor suppressors $p 21$ and NUMB (3,9-11), and inhibits their translation, subsequently resulting in the development of various types of cancer $(3,11,12)$. Together, these reports indicate that MSI2 plays an important role in the development and progression of various cancers and that MSI2 is a potential target of a novel anticancer therapeutic approach.

Ovarian cancer is one of the most fatal gynecological malignancies worldwide. The International Agency for Research on Cancer reported that in 2008, 225,000 new cases of ovarian cancer were diagnosed and that 140,000 patients died from the disease worldwide $(13,14)$. In Asian countries, there were an estimated 110,526 new cases of ovarian cancer diagnosed and 65,668 deaths from ovarian cancer in 2012 (14). As there are few distinguishing symptoms and etiological 
factors associated with the early stages of ovarian cancer (15), over $70 \%$ of cases are diagnosed at advanced metastatic stages (stage III or IV), a stage at which the 5-year survival rate is only $30 \%$ (16). Surgical debulking of tumors is the preferred initial treatment; however, approximately $80-90 \%$ of patients who undergo this procedure relapse in a relatively short period (17). The current standard of care consists of surgery followed by paclitaxel-based chemotherapy (18), despite the observation that patients who frequently relapse develop chemoresistance (19). It has been reported that chemoresistance is responsible for $90 \%$ of deaths in patients with advanced ovarian cancer (20), indicating that chemoresistance is the primary cause of ovarian cancer relapse. Although there have been considerable efforts to elucidate the mechanism of chemoresistance, molecular mechanisms underlying ovarian cancer relapse remain largely unknown. Therefore, we investigated the function of MSI2 in tumor growth and paclitaxel-resistance in ovarian cancer.

\section{Materials and methods}

Cell culture and reagents. The A2780 human ovarian cancer cell line was purchased from the European Collection of Cell Culture (Salisbury, UK). HeyA8, SKOV3, paclitaxel-resistant SKOV3-TR and immortalized ovarian surface epithelial (IOSE) cells were kindly provided by Dr Anil K. Sood (The University of Texas MD Anderson Cancer Center, Houston, TX, USA) and maintained in RPMI-1640 medium (Thermo Fisher Scientific, Waltham, MA, USA) supplemented with $10 \%$ (v/v) fetal bovine serum (FBS; Sigma-Aldrich, St. Louis, MO, USA) in a humidified atmosphere of $95 \%$ air and $5 \% \mathrm{CO}_{2}$ at $37^{\circ} \mathrm{C}$. Paclitaxel was purchased from Sigma-Aldrich and dissolved in dimethyl sulfoxide (DMSO; Sigma-Aldrich). The cells were labeled for quantitative analysis using $0.4 \%$ trypan blue (Gibco-Thermo Fisher Scientific).

Water-soluble tetrazolium salt (WST-1) assay. Paclitaxelinduced cytotoxicity was assessed using a WST-1 assay (EZ-Cytox cell viability assay kit; ITSBio, Seoul, Korea). The cells were seeded in a 96-well culture plate and incubated overnight. Then, the cells were treated with the indicated doses of paclitaxel for $48 \mathrm{~h}$; WST-1 solution was subsequently added to each well and the cells were incubated for $0.5 \mathrm{~h}$. Cell viability was determined by measuring absorbance at $450 \mathrm{~nm}$ using an iMark microplate reader (Bio-Rad Laboratories, Hercules, CA, USA).

BrdU incorporation assay. The rate of cell proliferation was evaluated using the BrdU Cell Proliferation Assay kit (Cell Signaling Technology, Danvers, MA, USA) according to the manufacturer's instructions. Briefly, the cells were transfected with control or MSI2-expressing plasmids and incubated overnight. The transfected cells were subsequently treated with $0.5 \%$ trypsin-EDTA (Gibco-Thermo Fisher Scientific). Then, $0.5 \times 10^{4}$ cells were added to each well of the 96-well plate; the cells were then incubated for $24 \mathrm{~h}$ and subsequently incubated in BrdU solution (1:10; Cell Signaling Technology) for $2 \mathrm{~h}$. Following the incubation, the cells were washed, treated with a fixing/denaturing solution (Cell Signaling Technology), and incubated with an antibody detection solution (Cell Signaling Technology) at room temperature for $1 \mathrm{~h}$. Then, the cells were washed, incubated in horseradish peroxidase (HRP)-conjugated second antibody solution (Cell Signaling Technology) for $30 \mathrm{~min}$, and incubated in TMB substrate solution (Cell Signaling Technology) for $30 \mathrm{~min}$. At the end of the incubation, cell proliferation was determined by measuring absorbance at $450 \mathrm{~nm}$ using an iMark microplate reader (BioRad Laboratories).

Immunoblot assay. Changes in protein levels were measured using immunoblotting assays. The cells were lysed in a radioimmunoprecipitation assay lysis buffer (Thermo Fisher Scientific) supplemented with a protease inhibitor cocktail (Roche Diagnostics, Indianapolis, IN, USA). The proteins were separated using SDS-PAGE and subsequently transferred to a nitrocellulose membrane (Bio-Rad Laboratories). The membrane was blocked with $5 \%$ skim milk for $1 \mathrm{~h}$ at room temperature and incubated overnight with the indicated antibody at $4^{\circ} \mathrm{C}$. HRP-conjugated anti-mouse or anti-rabbit secondary antibodies (Cell Signaling Technology) were used, followed by enhanced chemiluminescence (ECL; Pierce, Thermo Fisher Scientific) and autoradiography, to detect the proteins. The primary antibody against MSI2 was purchased from Abcam (Cambridge, UK). Anti-MDR1 and anti-Myc tag antibodies were purchased from Cell Signaling Technology. $\beta$-actin was used as a loading control and was detected using an antibody against it (Sigma-Aldrich).

Quantitative real-time PCR ( $q R T-P C R)$. Changes in mRNA levels were measured using qRT-PCR. The cells were lysed using TRIzol reagent (Invitrogen-Thermo Fisher Scientific), and total RNA was purified according to the manufacturer's instructions. cDNA was synthesized using M-MLV reverse transcriptase (Invitrogen-Thermo Fisher Scientific). PCR was performed using HOT FIREPol EvaGreen qPCR Mix Plus (Solis BioDyne, Tartu, Estonia) with a StepOnePlus Real-Time PCR System (Applied Biosystems-Thermo Fisher Scientific). The primers used to amplify human MSI2 were 5'-GTTAT CTGCGAACACAGTAGT-3' (forward) and 5'-CTCTGTGCC TGTTGGTAG-3' (reverse). The primers used to amplify human $M D R 1$ were 5'-TGACATTTATTCAAAGTTAAA GCA-3' (forward) and 5'-TAGACACTTTATGCAAACATT TCA-3' (reverse). The primers used to amplify human $\beta$-actin were 5'-GGATTCCTATGTGGGCGACGA-3' (forward) and 5'-CGCTCGGTGAGGATCTTCATG-3' (reverse). The $\mathrm{C}_{\mathrm{T}}$-values for MSI2 and MDR1 were normalized to $\beta$-actin. The $2^{-\Delta \Delta \mathrm{Ct}}$ method was used to calculate the relative expression levels of MSI2 and MDRI.

Transfection and lentiviral stable RNA interference. The human MSI2 open reading frame (ORF) was cloned into the pcDNA3.1-Myc/His vector (Invitrogen-Thermo Fisher Scientific), and the cells were transfected into the plasmids using Lipofectamine ${ }^{\circledR}$ (Invitrogen-Thermo Fisher Scientific). The MSI2 and scramble siRNAs were purchased from Bioneer Corp. (Daejeon, Korea), and the cells were transfected with siRNA using Lipofectamine ${ }^{\circledR}$ RNAiMAX (Invitrogen-Thermo Fisher Scientific). To generate the MSI2 lentiviral plasmid, ORF of MSI2 was cloned into the pLentiHT vector. The lentiviral vectors expressing shRNA against MSI2 were purchased from Sigma-Aldrich. To generate MSI2 ORF and MSI2 
A

\begin{tabular}{lcccccc}
\hline & & \multicolumn{5}{c}{ Immunohistochemical staining $n,(\%)$} \\
\cline { 3 - 8 } \multicolumn{1}{c}{ Histology } & No.of cases & Weak/No & $\%$ & Strong/Moderate & $\%$ & $P$ value \\
\hline Mucinous & 3 & 1 & 33.33 & 2 & 66.66 & \\
Serous & 16 & 1 & 6.25 & 15 & 93.75 & ${ }^{* *} 0.001$ \\
Undiffereniated & 4 & 0 & 0.00 & 4 & 100.00 & \\
Mullerian & 2 & 1 & 50.00 & 1 & 50.00 & \\
Endometrioid & 5 & 1 & 20.00 & 4 & 80.00 & \\
Clear cell & 5 & 2 & 40.00 & 3 & 60.00 & \\
others & 23 & 4 & 17.39 & 19 & 82.60 & \\
Total & 58 & 10 & 17.24 & 48 & 83.75 & ${ }^{*} 0.05$ \\
\hline
\end{tabular}
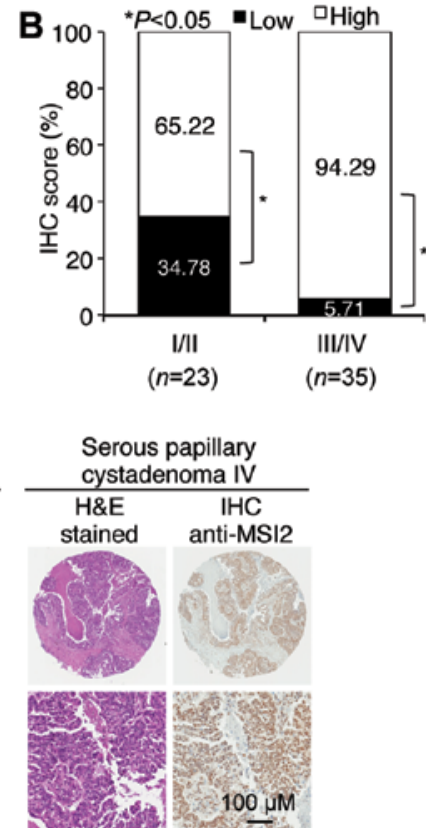

Figure 1. MSI2 protein expression in ovarian cancer tissues. (A) Immunohistochemistry (IHC) analysis of MSI2 in ovarian cancer tissues using an antibody against MSI2. No indicated IHC score 0 , weak indicated IHC score 1 , moderate indicated IHC score 2 , and strong indicated IHC score 3 . Statistical analysis were performed by the $\chi^{2}$ test, ${ }^{*} \mathrm{P}<0.05,{ }^{* *} \mathrm{P}<0.001$ was considered significant. (B) The IHC staining intensity of MSI 2 according to cancer stage. Low indicates a score of 0 or 1 and high indicates a score of 2 or $3 .{ }^{*} \mathrm{P}<0.05$ compared with high staining intensity scores. (C) MSI 2 protein expression levels in serous ovarian cancer tissues. H\&E, hematoxylin and eosin staining.

shRNA lentiviral particles, the 293T cells were co-transfected with the pCMV-VSV-G plasmid (Addgene, Cambridge, MA, USA) and pCMV-dR 8.2 (Addgene), and the viral particles were harvested after $72 \mathrm{~h}$. The cells were infected in the presence of polybrene $(8 \mu \mathrm{g} / \mathrm{ml})$. The cells were subsequently transduced and selected using $1 \mu \mathrm{g} / \mathrm{ml}$ puromycin (Amresco, Solon, OH, USA).

Annexin V/PI apoptosis assays. To analyze apoptosis, the cells were washed with phosphate-buffered saline (PBS; Thermo Fisher Scientific) and resuspended in 1X binding buffer (100 mM HEPES, pH 7.4, $140 \mathrm{mM} \mathrm{NaOH,} 2.5 \mathrm{mM}$ $\mathrm{CaCl}_{2}$ ). The cells were incubated with Annexin V-FITC and PI staining solution (FITC Annexin V apoptosis detection kit; BD Biosciences, Franklin Lakes, NJ, USA) for $15 \mathrm{~min}$ at RT in the dark. The quantity of apoptotic cells was determined using a BD FACSCalibur (BD Biosciences), and the results were analyzed using the CellQuest program (BD Biosciences).

Tissue immunohistochemistry (IHC) analysis. The expression level of MSI2 in ovarian cancer tissue samples was analyzed using ovarian cancer tissue microarrays (58 cases) (Super BioChips, Seoul, Korea). The tissue array was deparaffinized using xylene (Thermo Fisher Scientific) and rehydrated using graded ethanol $(100,95,90,80$ and $70 \%)$. After the slide was washed with distilled water, H\&E staining was performed using a Mayer's hamalum solution (Merck-Millipore, Billerica, MA, USA) and an Eosin Y-solution 0.5\%, aqueous (MerckMillipore). The stained slide was then dehydrated using graded ethanol (70, 90, 95 and 100\%) and xylene (Thermo Fisher Scientific), and the staining was visualized under a microscope. For IHC analysis, the ovarian cancer tissues were first incubated with a normal goat serum blocking solution
(2\% goat serum) and then stained for MSI2 by incubating with the MSI2-antibody (Abcam) diluted 1:100 overnight at $4^{\circ} \mathrm{C}$. The slide was washed with PBS supplemented with Tween-20 (0.05\% PBST) and subsequently incubated with HRP-conjugated secondary antibody diluted 1:5,000 for $90 \mathrm{~min}$ at room temperature. After the slides were washed in PBST, staining was visualized using a DAB substrate (Thermo Fisher Scientific). MSI2-positive cells were counted by a pathologist (Dr Yi Kyeong Chun, Cheil General Hospital and Women's Healthcare Center, Dankook University, College of Medicine, Seoul, Korea). Staining intensity was scored as follows: 0 , no staining; 1 , weak staining; 2 , moderate staining; and 3 , strong staining.

Statistical analysis. The results of three experiments per group were statistically analyzed. The data are presented as the mean \pm standard deviation (SD). Statistical analyses were conducted using a two-tailed Student's t-test analysis, chi-square test, or Fisher's exact test and Spearman's rank correlation coefficient analysis. $\mathrm{P}<0.05$ was considered statistically significant.

\section{Results}

MSI2 expression in ovarian cancer tissues. To establish the clinical significance of MSI2, we examined MSI2 expression in ovarian cancer tissues. Using a tissue microarray comprising 58 human ovarian cancer tissues, we conducted an IHC analysis of MSI2 expression and analyzed the correlation between MSI2 expression and various clinicopathological parameters such as tumor stage and subtype. In addition to being the most common histological subtype, serous ovarian cancer is the most lethal type of ovarian cancer, followed by 

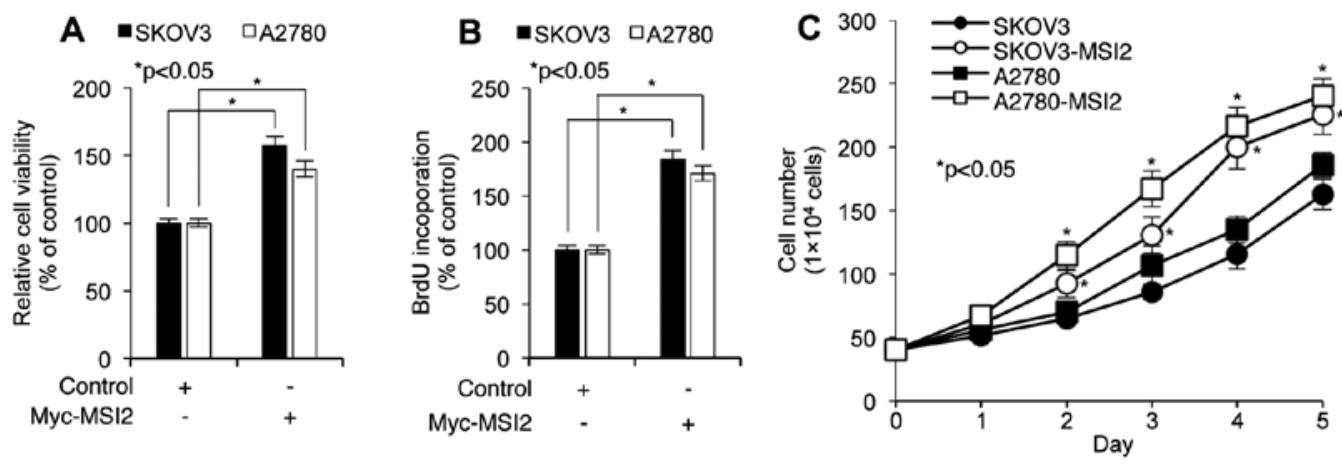

Figure 2. MSI2 promotes cell growth in ovarian cancer cells. (A) MSI2 overexpression enhances cell viability in ovarian cancer cells. SKOV3 and A2780 cells were transfected with a control or MSI2-expressing plasmid for $24 \mathrm{~h}$, and a WST-1-based cytotoxic assay was used to evaluate changes in cell viability. (B) MSI2 overexpression enhances cell proliferation in ovarian cancer cells. The ovarian cancer cells were transfected with a control or MSI2-expressing plasmid and subsequently analyzed using a BrdU incorporation assay. (C) MSI2 overexpression enhances cell growth in ovarian cancer cells. The number of cells was calculated daily for 5 days using a trypan blue staining assay. Data are presented as the mean \pm SD of three independent experiments. "P $<0.05$ compared with the corresponding DMSO-treated control cells.

A

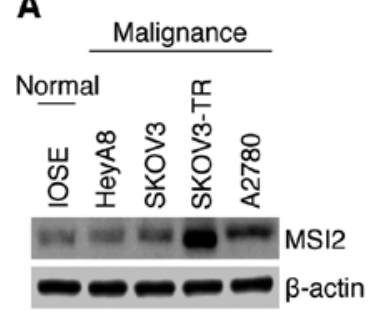

B

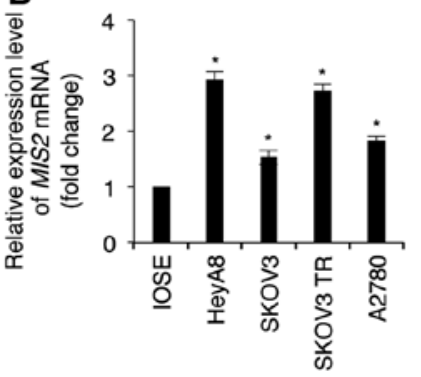

C "IOSE 口HеуA8 वSKOV3 -SKOV3-TR वA2780

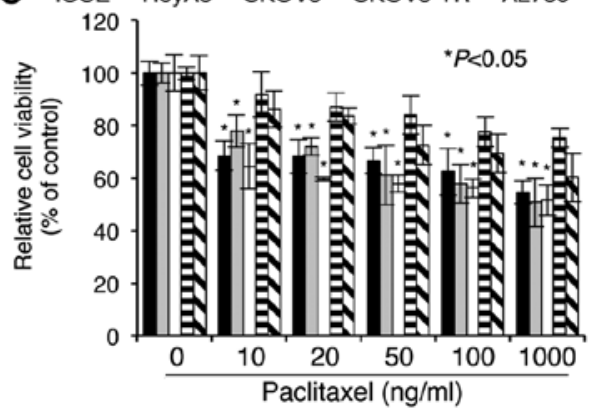

Figure 3. Expression of MSI2 in paclitaxel-sensitive and -resistant ovarian cancer cells. (A) MSI2 protein levels in ovarian cancer cells were evaluated using an immunoblotting assay with an antibody against MSI2. (B) MSI2 mRNA expression levels in ovarian cancer cells were evaluated using qRT-PCR. ${ }^{*} \mathrm{P}<0.05$ compared with the MSI2 level in ISOE cells. (C) The cytotoxic effect of paclitaxel on ovarian cancer cells. The cells were treated with the indicated doses of paclitaxel for $48 \mathrm{~h}$, and cell viability was evaluated using a WST-1 assay. ${ }^{*} \mathrm{P}<0.05$ compared with the cell viability of the corresponding cells not treated with paclitaxel. Data are presented as the mean \pm SD of three independent experiments.

mucinous, endometrioid and clear cell carcinoma (21). Our IHC results indicated that MSI2 was expressed at significantly higher levels in serous ovarian carcinoma tissues $(\mathrm{P}<0.001)$ than in other types of ovarian carcinoma tissues (Fig. 1A). In addition, MSI2 expression levels in advanced ovarian cancer tissues (grade III and IV) were significantly greater than in grade I and II tissues $(\mathrm{P}<0.05$; Fig. $1 \mathrm{~B}$ and $\mathrm{C})$. Together, these data suggested that MSI2 plays an important role in ovarian cancer growth and progression.

MSI2 overexpression accelerates the rate of ovarian cancer cell growth. Previous studies have shown that MSI2 regulates cell growth in several types of cancers including colorectal and brain cancers $(3,22)$. Therefore, we hypothesized that MSI2 affects the growth and proliferation in ovarian cancer cells. To address this hypothesis, we transfected MSI2 plasmids into ovarian cancer SKOV3 and A2780 cells and conducted a WST-1-based cell viability assay. As shown in Fig. 2A, cell viability significantly increased in cells overexpressing MSI2 than in the control cells. We further analyzed cell proliferation in the control and MSI2-transfected SKOV3 and A2780 cells using the BrdU incorporation assay. Consistent with the results of the cell viability assay, MSI2 overexpression significantly increased cell proliferation (Fig. 2B). We confirmed the results of the cell proliferation and viability assays using a cell growth assay. The control and MSI2-transfected cells were cultured for 5 days, and the relative cell numbers were evaluated daily using trypan blue staining. As expected, MSI2-overexpressing SKOV3 and A2780 cells exhibited a significant increase in growth rate compared with the control cells (Fig. 2C). Together, these results suggested that inhibiting MSI2 expression is a promising strategy for regulating ovarian cancer growth.

MSI2 is a novel mediator of paclitaxel sensitivity in ovarian cancer cells. Approximately $70 \%$ of patients with ovarian cancer are diagnosed at an advanced stage, and a majority of patients who initially respond to treatment ultimately relapse (23), which is commonly due to the development of chemoresistance (24). We found that MSI2 expression levels were significantly greater in stage III and IV ovarian cancer tissues than in stage tumor I and II tissues (Fig. 1) and that MSI2 overexpression enhanced ovarian cancer cell growth (Fig. 2). Therefore, we sought to determine if MSI2 also plays a role in paclitaxel resistance. To this end, we evaluated the expression levels of MSI2 in IOSE ovarian epithelial cells and various paclitaxel-sensitive and -resistant ovarian cancer cell lines 


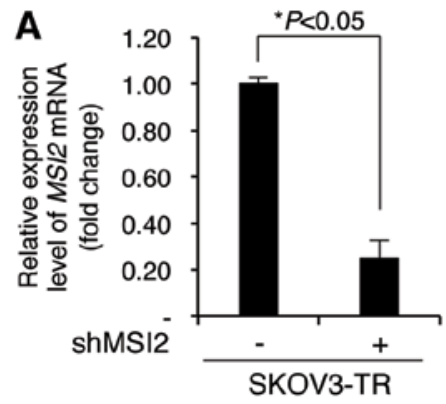

C

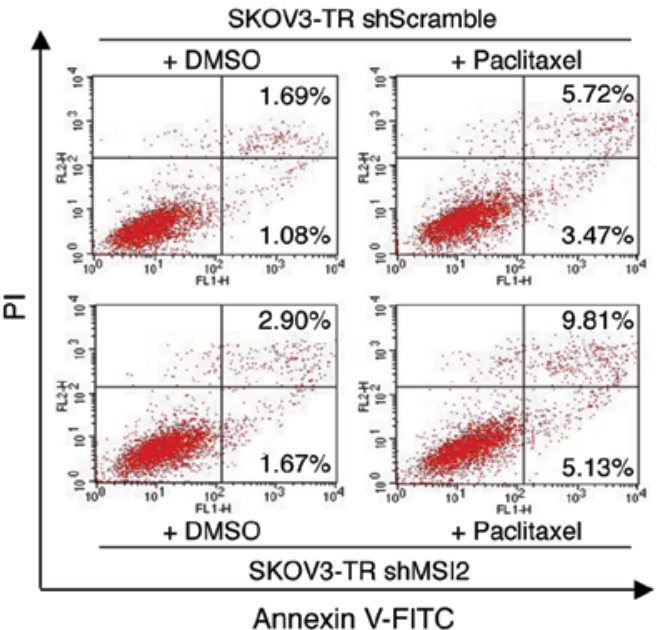

B

shMSI2 - +

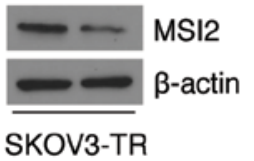

D
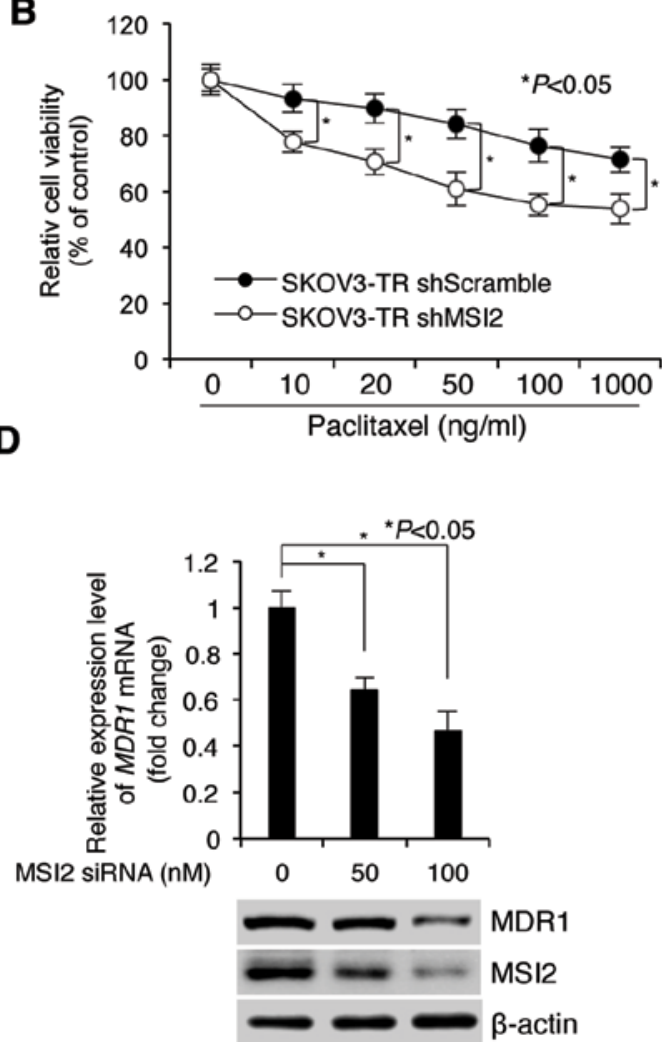

Figure 4. MSI2 knockdown induces paclitaxel sensitivity in paclitaxel-resistant ovarian cancer cells. (A) Lentivirus-mediated MSI2 knockdown in SKOV3-TR cells. Cells infected with the shMSI2 lentivirus were selected using G418-supplemented media. MSI2 mRNA and protein levels were analyzed using qRT-PCR and immunoblotting assays, respectively. The Student's t-test was used to calculate statistical significance ( $(\mathrm{P}<0.05)$. (B) MSI2 knockdown enhances paclitaxelinduced toxicity in SKOV3-TR cells. Control cells and MSI2 knockdown cells were treated with the indicated doses of paclitaxel for $48 \mathrm{~h}$. Cell viability was assessed using a WST-1 assay. The Student's t-test was used to calculate statistical significance ( $\left.{ }^{*} \mathrm{P}<0.05\right)$. (C) MSI2 knockdown increases paclitaxel-induced apoptosis in SKOV3-TR cells. Apoptosis was assessed using PI and Annexin V staining followed by flow cytometry. (D) MSI2 knockdown reduced MDR1 protein levels in SKOV3-TR cells. Cells were transfected with shRNA targeting MSI2, and MDR1 mRNA and protein levels were subsequently analyzed using qRT-PCR and immunoblotting assays, respectively. qRT-PCR data are presented as the mean \pm SD of three independent experiments. "P $<0.05$ compared with the corresponding DMSO-treated control cells.

(HeyA8, SKOV3, SKOV3-TR and A2780). To evaluate MSI2 protein levels, the cultured cells were lysed and analyzed using SDS-PAGE and immunoblotting with an MSI2-specific antibody. As shown in Fig. 3A, MSI2 protein levels were the highest in the paclitaxel-resistant SKOV3-TR cell line compared to the other cell lines. Moderate MSI2 levels were observed in A2780 cells, and relatively lower levels were observed in the IOSE, HeyA8 and SKOV3 cells. Next, we investigated MSI2 mRNA levels using qRT-PCR and found that MSI2 mRNA was highly expressed in the SKOV3-TR cells (Fig. 3B). However, the overall pattern of MSI2 mRNA expression was not identical to that of MSI2 protein expression, suggesting that post-transcriptional regulation influences the pattern of MSI2 expression in ovarian cancer cells. To further analyze the relationship between MSI2 protein levels and paclitaxel sensitivity in ovarian cancer cells, we conducted a WST-1-based cytotoxicity assay in the paclitaxel-treated cells. As shown in Fig. 1C, the SKOV3-TR cells, which exhibited elevated MSI2 protein levels, exhibited the strongest paclitaxel resistance at all doses examined. The A2780 cells, which exhibited only moderate MSI2 protein levels, exhibited moderate paclitaxel resistance at low doses $(<20 \mathrm{ng} / \mathrm{ml})$; however, higher doses of paclitaxel were significantly cytotoxic. Ovarian cancer cells with low MSI2 protein levels (IOSE, HeyA8 and SKOV3) exhibited paclitaxel sensitivity even at low doses (Fig. 3C). These data suggested that paclitaxel sensitivity and resistance are regulated by MSI2 protein levels in ovarian cancer cells.

MSI2 depletion increases paclitaxel sensitivity in paclitaxelresistant SKOV3-TR cells. Based on the results described above, we hypothesized that MSI2 regulates paclitaxel cytotoxicity in ovarian cancer cells. To investigate this hypothesis, we generated stable MSI2 knockdown cells using an shRNA-expressing lentivirus. As shown in Fig. 4A, we observed a significant reduction in MSI2 mRNA and protein levels in shMSI2-expressing SKOV3-TR cells. In addition, paclitaxel exerted a significantly greater toxicity in the shMSI2expressing SKOV3-TR cells than in the corresponding control cells (SKOV3-TR shScramble) (Fig. 4B), indicating MSI2 knockdown abolished paclitaxel resistance in the SKOV3-TR cells. To confirm these data, we evaluated apoptosis in the control and MSI2-knockdown SKOV3-TR cells treated with paclitaxel. Consistent with the results of the cytotoxicity assays, paclitaxel-induced apoptosis (both early and late apoptosis) increased in the MSI2-knockdown SKOV3-TR cells compared with that in the control SKOV3-TR cells (Fig. 4C). Previous reports have demonstrated that paclitaxel resistance is correlated with multidrug resistant 1 (MDR1) expression 
A

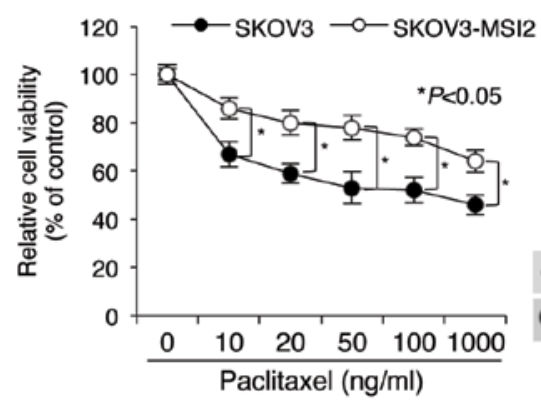

C

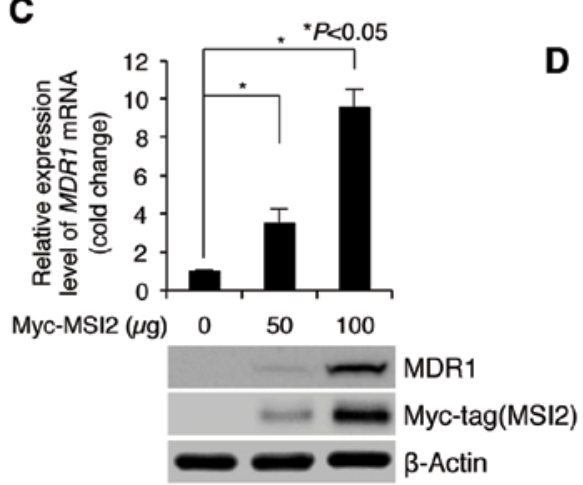

B

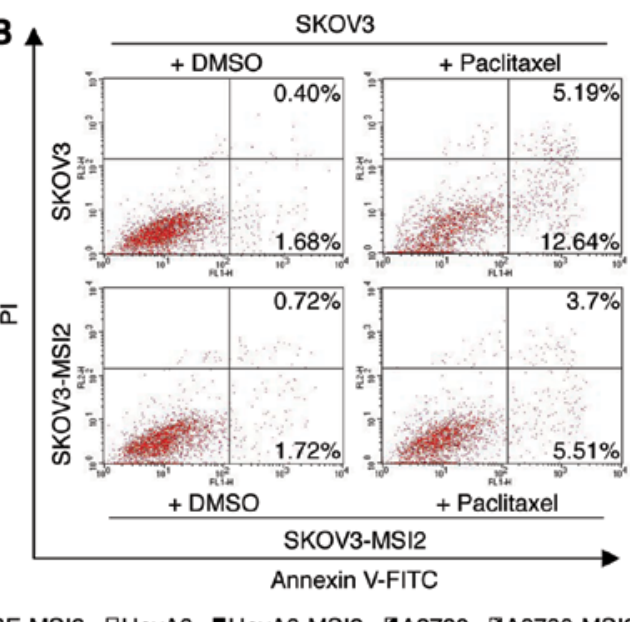

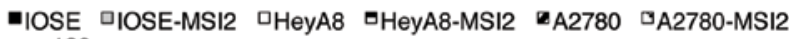

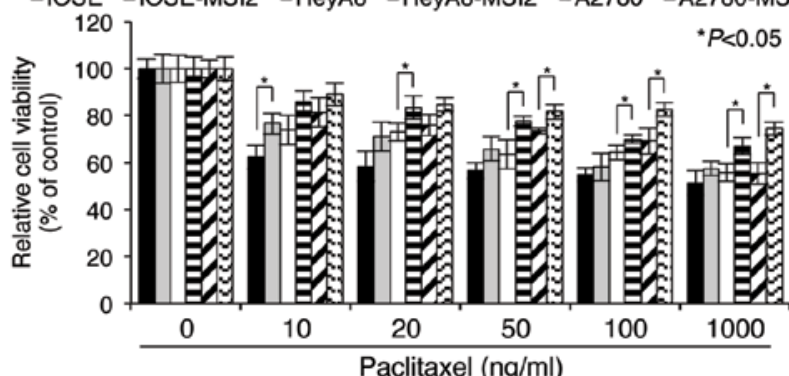

Figure 5. MSI2 overexpression increases paclitaxel resistance in ovarian cancer cells. (A) MSI2 overexpression inhibits paclitaxel toxicity in SKOV3 cells. SKOV3 cells were treated with the control or MSI2-expressing lentivirus and subsequently treated with the indicated doses of paclitaxel. Cell viability was analyzed using a WST-1 assay. The Student's t-test was used to evaluate statistical significance ( $\left.{ }^{*}<0.05\right)$. (B) MSI2 overexpression inhibits paclitaxel-induced apoptosis in SKOV3 cells. Control and MSI2-overexpressing cells were treated with paclitaxel, and apoptosis was assessed using PI and Annexin V staining followed by flow cytometry. (C) MSI2 overexpression increases MDR1 expression levels in SKOV3 cells. Cells were transfected with myc-tagged MSI2 plasmids, and MDR1 mRNA and protein levels were analyzed using qRT-PCR and immunoblotting assays, respectively. qRT-PCR data are presented as the mean \pm SD of three independent experiments. ${ }^{*} \mathrm{P}<0.05$ compared with the corresponding DMSO-treated control cells. (D) MSI 2 overexpression inhibits paclitaxel toxicity in ovarian cancer cells. HeyA8 and A2780 cells were transfected with myc-tagged MSI2 plasmids and subsequently treated with the indicated doses of paclitaxel. Cell viability was evaluated using a WST- 1 assay. Data are presented as the mean \pm SD of three independent experiments. ${ }^{*}<0.05$ compared with the corresponding control and paclitaxel-treated control cells.

levels (25-27). To determine if shMSI2-induced paclitaxel sensitivity was associated with changes in MDR1 levels, we evaluated MDR1 levels in the cells transfected with MSI2specific siRNA using qRT-PCR and immunoblotting assays. As shown in Fig. 4D, MDRl mRNA and protein levels significantly decreased in the SKOV3-TR cells transfected with MSI2 siRNA in a dose-dependent manner. Together, these data suggested that MSI2 plays an important role in paclitaxel resistance in ovarian cancer cells.

MSI2 overexpression induces paclitaxel resistance in ovarian cancer cells. Next, we examined if MSI2 overexpression can induce paclitaxel resistance in paclitaxel-sensitive SKOV3 cells. To this end, we generated stable MSI2-overexpressing SKOV3 (SKOV3-MSI2) cells using an MSI2-expressing lentivirus (Fig. 5A, right panel) and analyzed their cell viability after paclitaxel treatment. As shown in Fig. 5A, the paclitaxel-mediated inhibition of cell viability significantly decreased in the MSI2-overexpressing SKOV3 cells than in the control SKOV3 cells. To further evaluate the inhibitory effect of MSI2 on paclitaxel-induced apoptosis, we treated the control and MSI2-overexpressing SKOV3 cells with paclitaxel and analyzed apoptosis using Annexin V/PI staining and flow cytometry. As shown in Fig. 5B, paclitaxel-induced apoptosis significantly decreased in the MSI2-overexpressing SKOV3 cells than in the control SKOV3 cells. As siRNA directed against MSI2 inhibited MDR1 expression in the SKOV3-TR cells (Fig. 4D), we investigated the relationship between MSI2-mediated paclitaxel resistance and MDR1 expression in the SKOV3 cells. As expected, transient MSI2 overexpression upregulated MDR $1 \mathrm{mRNA}$ and protein levels in the SKOV3 cells in a dose-dependent manner (Fig. 5C). Finally, we sought to determine if the effect of MSI2 on paclitaxel resistance in the SKOV3-TR cells was similar to that in other ovarian cancer cells (HeyA8 and A2780). Therefore, we used the WST-1 assay to evaluate cell viability in the MSI2-overexpressing HeyA8 and A2780 cells treated with the indicated doses of paclitaxel for $48 \mathrm{~h}$. Consistent with that observed in the SKOV3 cells, paclitaxel resistance increased in the MSI2-expressing HeyA8 and A2780 cells compared with that in the corresponding non-transfected control cells (Fig. 5D). Together, these data indicate that MSI2 is a novel contributor to paclitaxel resistance in ovarian cancer cells.

\section{Discussion}

The results of the present study describe two novel functions of MSI2 in ovarian cancer and provide the first evidence 
suggesting that MSI2 plays an important role in the development of ovarian cancer. Tissue microarray experiments revealed that MSI2 expression was significantly correlated with advanced (III-IV), metastatic ovarian cancer and that elevated MSI2 expression levels are correlated with serous ovarian carcinoma tissues. We also demonstrated that transient MSI2 overexpression strongly increased cell viability, proliferation, and growth in the SKOV3 and A2780 cells, which are two established models of serous ovarian carcinoma. The most common type of ovarian cancer is epithelial ovarian cancer, of which serous ovarian carcinoma is the most commonly diagnosed subtype (16). Indeed, approximately $80-85 \%$ of cases of ovarian carcinomas in the Western world are of the serous subtype, and up to $95 \%$ of patients with advanced ovarian cancer are diagnosed with serous carcinomas (28). Because serous carcinoma is the most prevalent subtype, it is likely that previous clinical and molecular studies on ovarian cancer highly represent cases of serous carcinomas (16). In other types of cancers, MSI2 has been considered a promising therapeutic target due to its anti-apoptotic and oncogenic functions and its correlation with poor prognosis $(4,7,11)$. Therefore, our finding that MSI2 expression is elevated in advanced, serous ovarian cancer tissues might provide novel insights into the study of ovarian cancer. Although the molecular mechanisms that regulate MSI2 in ovarian cancer and the association of MSI2 expression with other clinicopathological features of ovarian cancer remain unknown, the findings of this study merit further investigation.

Paclitaxel, a derivative of the chemotherapeutic drug taxane, plays a key role in the treatment of ovarian cancer (16). Although up to $42 \%$ of patients with ovarian cancer initially respond to paclitaxel treatment, most patients ultimately experience disease relapse and become chemoresistant (29). Currently, ovarian cancer is primarily diagnosed at advanced stages; therefore, overcoming the challenge of paclitaxel resistance is essential in prolonging overall survival. Our data further suggest that MSI2 induces paclitaxel resistance in ovarian cancer cells. This hypothesis is supported by the observation that MSI2 is expressed at significantly higher levels in the paclitaxel-resistant SKOV3 cells than in the paclitaxel-sensitive cells. In addition, we demonstrated that the incidence of paclitaxel-induced cell death increased in the MSI2-depleted SKOV3-TR cells compared with that in the control SKOV3-TR cells. In addition, MSI2 overexpression induced paclitaxel resistance in the paclitaxel-sensitive ovarian cancer cells. These data suggest that MSI2 plays an essential role in paclitaxel resistance and that high levels of MSI2 expression might correlate with paclitaxel resistance in ovarian cancer. Consistent with our results, recent reports have shown that MSI2 is associated with the development of chemoresistance in other types of cancer. One report has demonstrated that MSI2-expressing cells are more tumorigenic than non-MSI2-expressing cells and that MSI2 promotes resistance to anticancer drugs in pancreatic cancer cells (30). Another report has demonstrated that the loss of MSI2 inhibits tumor cell proliferation and sensitizes AML cells to anticancer drugs (12). Finally, we found that MSI2 knockdown decreased MDR1 expression levels in the SKOV3-TR cells, whereas transient MSI2 overexpression increased MDR1 expression levels. Together, these findings suggest that MSI2 is a novel regulator of MDR1. MDR1, also referred to as P-glycoprotein (ABCB1), is an important regulator of chemoresistance that is overexpressed in various drug-resistant cancer cells such as paclitaxel-resistant cells (31). siRNA-induced MDR1 knockdown significantly sensitized SKOV3-TR and OVCAR8-TR cells to paclitaxel (26). These results indicate that the molecular functions of MSI2 and MDR1 chemoresistance in ovarian cancer cells are related. Further investigation into the mechanism underlying the interplay between MSI2 and MDR1 might provide new insights into the resistance of cancer cells to anticancer drugs.

\section{Acknowledgements}

The pressent study was supported by the KU Research Professor Program of Konkuk University (to S.B.) and the National Research Foundation of Korea (NRF) grants from the Ministry of Science, ICT and Future Planning (grant 2013R1A1A1012205 to S.B., and grant 20110028646 to S.A.).

\section{References}

1. Okano H, Kawahara H, Toriya M, Nakao K, Shibata S and Imai T: Function of RNA-binding protein Musashi-1 in stem cells. Exp Cell Res 306: 349-356, 2005.

2. Sutherland JM, McLaughlin EA, Hime GR and Siddall NA: The Musashi family of RNA binding proteins: Master regulators of multiple stem cell populations. Adv Exp Med Biol 786: 233-245, 2013.

3. Wang S, Li N, Yousefi M, Nakauka-Ddamba A, Li F, Parada K, Rao S, Minuesa G, Katz Y, Gregory BD, et al: Transformation of the intestinal epithelium by the MSI2 RNA-binding protein. Nat Commun 6: 6517, 2015.

4. Kharas MG, Lengner CJ, Al-Shahrour F, Bullinger L, Ball B, Zaidi S, Morgan K, Tam W, Paktinat M, Okabe R, et al: Musashi-2 regulates normal hematopoiesis and promotes aggressive myeloid leukemia. Nat Med 16: 903-908, 2010.

5. Griner LN and Reuther GW: Aggressive myeloid leukemia formation is directed by the Musashi 2/Numb pathway. Cancer Biol Ther 10: 979-982, 2010.

6. Mu Q, Wang Y, Chen B, Qian W, Meng H, Tong H, Chen F, Ma Q, Ni W, Chen S, et al: High expression of Musashi-2 indicates poor prognosis in adult B-cell acute lymphoblastic leukemia. Leuk Res 37: 922-927, 2013

7. He L, Zhou X, Qu C, Hu L, Tang Y, Zhang Q, Liang M and Hong J: Musashi2 predicts poor prognosis and invasion in hepatocellular carcinoma by driving epithelial-mesenchymal transition. J Cell Mol Med 18: 49-58, 2014.

8. Sakakibara S, Nakamura Y, Satoh H and Okano H: RNA-binding protein Musashi2: Developmentally regulated expression in neural precursor cells and subpopulations of neurons in mammalian CNS. J Neurosci 21: 8091-8107, 2001.

9. Imai T, Tokunaga A, Yoshida T, Hashimoto M, Mikoshiba K, Weinmaster G, Nakafuku $M$ and Okano $H$ : The neural RNA-binding protein Musashil translationally regulates mammalian numb gene expression by interacting with its mRNA. Mol Cell Biol 21: 3888-3900, 2001

10. Battelli C, Nikopoulos GN, Mitchell JG and Verdi JM: The RNA-binding protein Musashi-1 regulates neural development through the translational repression of p21WAF-1. Mol Cell Neurosci 31: 85-96, 2006.

11. Zhang H, Tan S, Wang J, Chen S, Quan J, Xian J, Zhang S, He J and Zhang L: Musashi2 modulates K562 leukemic cell proliferation and apoptosis involving the MAPK pathway. Exp Cell Res 320: 119-127, 2014.

12. Han Y, Ye A, Zhang Y, Cai Z, Wang W, Sun L, Jiang S, Wu J, $\mathrm{Yu} \mathrm{K}$ and Zhang S: Musashi-2 silencing exerts potent activity against acute myeloid leukemia and enhances chemosensitivity to daunorubicin. PLoS One 10: e0136484, 2015.

13. Ferlay J, Shin HR, Bray F, Forman D, Mathers C and Parkin DM: Estimates of worldwide burden of cancer in 2008: GLOBOCAN 2008. Int J Cancer 127: 2893-2917, 2010. 
14. Razi S, Ghoncheh M, Mohammadian-Hafshejani A, Aziznejhad $\mathrm{H}$, Mohammadian $\mathrm{M}$ and Salehiniya $\mathrm{H}$ : The incidence and mortality of ovarian cancer and their relationship with the Human Development Index in Asia. E Cancer Med Sci 10: 628, 2016.

15. Jelovac D and Armstrong DK: Recent progress in the diagnosis and treatment of ovarian cancer. CA Cancer J Clin 61: 183-203, 2011.

16. Cho KR and Shih IeM: Ovarian cancer. Annu Rev Pathol 4: 287-313, 2009.

17. Schorge JO, McCann C and Del Carmen MG: Surgical debulking of ovarian cancer: What difference does it make? Rev Obstet Gynecol 3: 111-117, 2010.

18. Hennessy BT, Coleman RL and Markman M: Ovarian cancer. Lancet 374: 1371-1382, 2009.

19. McGuire WP and Ozols RF: Chemotherapy of advanced ovarian cancer. Semin Oncol 25: 340-348, 1998.

20. Sherman-Baust CA, Becker KG, Wood Iii WH, Zhang Y and Morin PJ: Gene expression and pathway analysis of ovarian cancer cells selected for resistance to cisplatin, paclitaxel, or doxorubicin. J Ovarian Res 4: 21, 2011.

21. Nakayama K, Nakayama N, Jinawath N, Salani R, Kurman RJ, Shih IeM and Wang TL: Amplicon profiles in ovarian serous carcinomas. Int J Cancer 120: 2613-2617, 2007.

22. Cox JL, Wilder PJ, Gilmore JM, Wuebben EL, Washburn MP and Rizzino A: The SOX2-interactome in brain cancer cells identifies the requirement of MSI2 and USP9X for the growth of brain tumor cells. PLoS One 8: e62857, 2013.

23. Ledermann JA and Kristeleit RS: Optimal treatment for relapsing ovarian cancer. Ann Oncol 21 (Suppl 7): vii218-vii222, 2010.
24. Zhan Q, Wang $C$ and Ngai S: Ovarian cancer stem cells: A new target for cancer therapy. BioMed Res Int 2013: 916819, 2013.

25. Mechetner E, Kyshtoobayeva A, Zonis S, Kim H, Stroup R, Garcia R, Parker RJ and Fruehauf JP: Levels of multidrug resistance (MDR1) P-glycoprotein expression by human breast cancer correlate with in vitro resistance to taxol and doxorubicin. Clin Cancer Res 4: 389-398, 1998.

26. Duan Z, Brakora KA and Seiden MV: Inhibition of ABCB (MDR1) and ABCB4 (MDR3) expression by small interfering RNA and reversal of paclitaxel resistance in human ovarian cancer cells. Mol Cancer Ther 3: 833-838, 2004.

27. Horwitz SB, Cohen D, Rao S, Ringel I, Shen HJ and Yang CP: Taxol: Mechanisms of action and resistance. J Natl Cancer Inst Monogr 15: 55-61, 1993

28. Colombo N, Peiretti M, Parma G, Lapresa M, Mancari R, Carinelli S, Sessa C and Castiglione M; ESMO Guidelines Working Group: Newly diagnosed and relapsed epithelial ovarian carcinoma: ESMO Clinical Practice Guidelines for diagnosis, treatment and follow-up. Ann Oncol 21 (Suppl 5): v23-v30, 2010.

29. Ozols RF: Recurrent ovarian cancer: Evidence-based treatment J Clin Oncol 20: 1161-1163, 2002.

30. Fox RG, Lytle NK, Jaquish DV, Park FD, Ito T, Bajaj J, Koechlein CS, Zimdahl B, Yano M, Kopp JL, et al: Image-based detection and targeting of therapy resistance in pancreatic adenocarcinoma. Nature 534: 407-411, 2016.

31. Goda K, Bacsó Z and Szabó G: Multidrug resistance through the spectacle of P-glycoprotein. Curr Cancer Drug Targets 9: 281-297, 2009. 